\title{
LA OCDE: UN PERFIL CRÍTICO
}

\author{
Felipe A. Galvis Castroa)
}

THE OECD: A CRITICAL PROFILE

A OCDE PERFIL CRÍTICO

Fecha de recepción: 3 de enero del 2020

Fecha de aprobación: 25 de junio del 2020

Disponible en línea: 25 de septiembre del 2020

\section{Sugerencia de citación:}

Galvis Castro, F. A. (2021). La ocDE: un perfil crítico. Razón Crítica, 10, 211-235. https://doi. org/10.21789/25007807.1568

(a) Felipe A. Galvis Castro

Ph. D. en Derecho, LL.M., en Derecho Internacional, Magíster en Derecho, abogado.

Profesor de la Universidad del Rosario, Pontificia Universidad Javeriana y Universitat Oberta de Catalunya

https:// orcid.org/0000-0002-1552-0602

fgalviscastro@gmail.com 


\section{R E S U M E N}

En este artículo se propone realizar una caracterización crítica de la Organización para la Cooperación y el Desarrollo Económicos (OCDE) a partir de su orientación general, tareas y medios empleados en el cumplimiento de sus funciones. Del mismo modo, se presenta una aproximación a la labor de esa Organización a partir de la utilización que hace de evidencia empírica y conocimiento experto en la identificación de las mejores prácticas de gobierno, en la precisión de estándares con vocación normativa, en la promoción de marcos regulatorios y en el impulso que ofrece a la formación de convergencias en políticas públicas. Esta contribución concluye con un análisis del compromiso de la ocDE con una determinada racionalidad rectora de política económica, así como con la promoción de un ideal de Estado y de sujeto.

PALABRAS CLAVE: derecho blando; gobernanza; neoliberalismo; OCDE, sujetos 


\section{A B S T R A C T}

This article presents a critical description of the Organization for Economic Cooperation and Development (OECD) based on the general orientation, tasks and means for the fulfillment of the functions of this organization. In the same way, an analysis to the work by the OECDD is presented taking into account how this international body uses empirical evidence and expert knowledge for the identification of best governance practices, the establishment of standards with a normative focus, the promotion of regulatory frameworks, and the impulse it offers to the creation of convergences in public policies. This work concludes with an analysis of the OECD's commitment to a specific rationale for driving economic development and the promotion of ideal States and individuals. Keywords: Soft law, governance, neoliberalism, OECD, subjects.

\section{PALABRAS CLAVES: soft law; governance; neoliberalism; OECD; subjects.}

\section{R E S U M O}

Neste artigo, propõe-se a realizar uma caracterização crítica da Organização para a Cooperação e Desenvolvimento Econômico (ocDE) a partir de sua orientação geral, das tarefas e dos meios utilizados no cumprimento de suas funções. Do mesmo modo, é apresentada uma aproximação do trabalho dessa organização a partir da utilização que ela faz de evidência empírica e conhecimento especializado na identificação das melhores práticas de governo na precisão de padrões com aptidão normativa, na promoção de referenciais regulatórios e no impulso que oferece à formação de convergências em políticas públicas. Esta contribuição conclui com uma análise do compromisso da OCDE com um determinado raciocínio desafiador de política econômica, bem como com a promoção de um ideal de Estado e de sujeito.

PALAVRAS-CHAVE: direito suave; soft law; governança; neoliberalismo; OCDE; sujeitos. 


\section{N T R O D U C C I Ó N}

No solo la ocDE ha sido poco estudiada, sino que la mayoría de la literatura existente sobre ella ha sido producida bajo el auspicio de su propia organización. Sin duda, la aproximación a ese tipo de documentos requiere de cierta distancia, de manera que se los considere parte del objeto de examen y no tanto una referencia valorativa del funcionamiento de esa institución internacional.

Por esta razón a continuación se presenta lo que podría considerarse un estado del arte crítico acerca del perfil y funcionamiento de la oCDE. De modo que, esta lectura permite equilibrar la comprensión de este importante actor global, entre una descripción más cercana a su visión institucional y un examen "desde afuera" de las principales líneas de actuación que permiten caracterizarla. Asimismo, esta aproximación hace posible advertir cómo la invocación de la oCDE como organización legitimadora de la inevitabilidad y de la convergencia global en materia de políticas públicas, naturaliza algunas de las inclinaciones generales que ha presentado esta institución internacional en su actuación.

Esta caracterización de la labor de la ocDE no tiene por objeto presentarla como una institución monolítica, con una orientación única para todas las materias, que ofrece las mismas fórmulas para todos los contextos y circunstancias. No obstante, sí pretende advertir cómo la ocDE está lejos de ser el espacio de promoción y divulgación de ideas formadas sobre la base de un conocimiento experto, técnicamente sustentadas y políticamente balanceadas (si es que eso existe), sobre las cuales se afirma de forma acrítica la convergencia de políticas. Precisamente esta aproximación acrítica ha dominado el análisis en el país durante 
los últimos años, a propósito de la reciente conclusión del proceso de admisión del Estado como miembro de esta organización.

En ese sentido, se observa que desde la década de 1970 la ocDE ha presentado una evidente orientación neoliberal, que ha sido reemplazada más recientemente por una aproximación de "liberalismo inclusivo". A pesar de que actualmente hay una mayor atención hacia asuntos directamente relacionados con el bienestar social de los ciudadanos, este impulso no ha implicado un abandono de los imperativos macroeconómicos bajo los cuales ha actuado esta organización desde la década de 1970

Con esta perspectiva, este artículo presenta a continuación: (a) el origen y la proyección global de la ocDE; (b) las tareas y los medios que emplea habitualmente; (c) las críticas que han surgido de acuerdo a la manera en que interpreta la evidencia existente acerca de las mejores políticas implementadas a nivel global; (d) la racionalidad rectora que predomina en ella, y (e) la forma en que esta se materializa en la promoción de una identidad de Estado moderno y de una subjetividad específica.

\section{ORIGEN Y PROYECGIÓN GLOBAL}

En el contexto de la gobernanza global contemporánea, la OCDE ha operado de forma persistente y muchas veces inadvertida sobre las condiciones en que gobiernos, sociedades e individuos dirigen sus vidas, se trazan objetivos, determinan medios para alcanzarlos y valoran su existencia. Para ello, la oCDE ha sido pionera en la utilización de medios de incidencia sobre las sociedades, que posteriormente han sido usados por organizaciones internacionales más visibles y tradicionales, como los ejercicios de revisión por pares o el uso de redes de políticas (Mahon \& McBride, 2008). Estos medios parten de comprender la importancia de la socialización entre actores con presencia internacional, como un aspecto decisivo en el seguimiento de los Estados a marcos regulatorios y modelos de políticas públicas, importancia destacada por Goodman y Jinks (2013).

Lo anterior posiciona a la OCDE como una de las instituciones que mejor interpreta el sentido y las formas de la gobernanza global contemporánea, en tanto que contribuye a articular una densa constelación de redes, actores e intereses (que definen conjuntamente 
modelos regulatorios) y asigna el prestigio de seguir las mejores prácticas de gobierno, aquellas implementadas por las "economías líderes".

En términos históricos, la ocDE surge a partir del proyecto de la Organización para la Cooperación Económica Europea (oEEc, por sus siglas en inglés), institución creada en 1948 como espacio de "consulta intra-europea" en el marco de la cooperación del Plan Marshall para la reconstrucción de parte del continente, que con el tiempo desarrolló una agenda de promoción de la cooperación, el libre comercio y la unión en materia arancelaria (Salzman, 2005; Leimgruber \& Schmelzer, 2017). La OEEC agrupaba en su primera década de existencia a los países europeos (con excepción de España), a los cuales se asociaron Estados Unidos y Canadá. Para 1958, todos los miembros de la oeEc eran al mismo tiempo miembros de la Organización del Tratado del Atlántico Norte (otan) (Wolfe, 2008).

Ante la firma del Tratado de Roma de 1957- con las perspectivas de crear un mercado común entre los seis países fundadores de la Comunidad Económica Europea (CEE), para 1958 se ponía en duda la importancia de la oEEc. En esa coyuntura se realizó una cumbre entre los gobiernos de Francia, Estados Unidos, República Federal de Alemania y el Reino Unido en París, en la que se lanzaron las negociaciones que dieron lugar a la constitución de la actual ocDE. La cumbre se inspiraba en la convicción de que "el imperativo político de la cohesión atlántica durante la Guerra Fría era tan importante como la cooperación en política económica"1 y se convino que se "destinasen esfuerzos a promover el progreso de los países menos desarrollados" (Wolfe, 2008, p. 27). En consecuencia, la ocDE se perfiló desde sus orígenes en 1960, como una organización principalmente “atlántica, no europea”, que debía establecer un diálogo con los países "menos desarrollados" (Wolfe, 2008, p. 27)², particularmente con

1 La ocDe se fundó entonces como parte de la alianza que se opondría al bloque socialista que forjó la Unión Soviética en Europa del Este. Más adelante, su departamento económico jugó un importante papel en el tránsito de los países que conformaban ese bloque, hacía economías de libre mercado en las décadas de 1980 y 1990, a propósito del examen de las políticas de esos países en el marco de "procesos de monitoreo". Entre los 1990 y los 2000 se integrarían como miembros de la ocDE: República Checa, Hungría, Polonia y Eslovaquia (Mahon \& McBride, 2009, p. 92). 2 Los objetivos declarados de la ocdese encuentran en el tratado constitutivo de la Organización, aprobado en París, el 14 de diciembre de 1960: "Artículo I. La Organización de Cooperación y de Desarrollo Económicos (llamada en adelante la Organización) tiene como objetivos el promover políticas destinadas a: (a) realizar la más fuerte expansión posible de la economía y del empleo y a un aumento del nivel de vida en los países miembros, manteniendo la estabilidad financiera y a contribuir así al desarrollo de la economía mundial; (b) contribuir a una sana expansión económica en los países miembros y en los no miembros en vías de desarrollo económico; (c) contribuir a la expansión del comercio mundial sobre una base multilateral y no discriminatoria, conforme a las obligaciones internacionales. // Artículo II. En la persecución 
Europa del Este y el Sur Global (Leimgruber \& Schmelzer, 2017). En sus inicios, la OCDE se pensó como una especie de “OTAN económica” y se caracterizó por promover los valores de desarrollo propios de "occidente" (Leimgruber \& Schmelzer, 2017, p. 43) ${ }^{3}$.

Desde esa inspiración noratlántica original, la OCDE ha comprendido especialmente a partir del fortalecimiento del proceso de integración europea y el surgimiento de relevantes foros como el G7, la posibilidad de caer en la irrelevancia, así como la importancia de extender su margen de influencia más allá de ese marco geopolítico original. Con el paso de los años, la OCDE “dedica más tiempo hablando a terceros países [no miembros] que el que ocupa a hablar acerca de ellos [miembros]" (Wolfe, 2008, p. 28). Así, la meta fundamental de la oCDE a finales del siglo xx y comienzos del Xxi ha sido "ampliar la zona de paz estable mediante la difusión del conocimiento acerca de cómo funciona la economía liberal hacia un número mayor de países" (Wolfe, 2008, p. 33). La organización ha desarrollado un proceso que paulatinamente le ha permitido ampliar su audiencia, incluyendo nuevos participantes y dirigiéndose al diálogo con Estados no miembros, interesados en atender sus recomendaciones ${ }^{4}$. En particular, esta organización ha mostrado especial deferencia en las últimas décadas hacia la audiencia que puede encontrar dentro de las economías más importantes de Asia y América Latina (Mahon \& McBride, 2009).

Ahora bien, en cuanto a América Latina, la ocDE lanzó en el 2016 un programa de cooperación para fortalecer los lazos y canales de intercambio con la Región. Lo cual resultó de especial interés para la oCDE, con tres miembros de la organización en esta región, México,

de esos objetivos, los miembros convienen que tanto en forma individual, como conjunta: (a) promoverán la utilización eficiente de sus recursos económicos; (b) en el terreno científico y técnico, promoverán el desarrollo de sus recursos, fomentarán la investigación y favorecerán la formación profesional; (c) perseguirán políticas diseñadas para lograr el crecimiento económico y la estabilidad financiera interna y externa y para evitar que aparezcan situaciones que pudieran poner en peligro su Economía o la de otros países; (d) continuarán los esfuerzos por reducir o suprimir los obstáculos a los intercambios de bienes y de servicios y a los pagos corrientes y por mantener y extender la liberalización de los movimientos de capital; (e) contribuirán al desarrollo económico tanto de los países miembros como de los no miembros en vías de desarrollo económico, a través de los medios apropiados, en particular a través de la afluencia de capitales a esos países, considerando la importancia que tiene para sus economías la asistencia técnica y el asegurar una ampliación de los mercados ofrecidos a sus productos de exportación" (OCDE, 1960, p. 2).

3 En un comienzo se consideraron nombres para esta institución como "Organización para la Cooperación Económica Atlántica y Organización Económica Atlántica” (Leimgruber \& Schmelzer, 2017, p. 47).

4 El número de países que han sido admitidos a la ocDE es amplio. Algunos ejemplos son Japón (1964), Australia (1973), Nueva Zelanda (1973), México (1994), Corea del Sur (1996), entre otros. 
Colombia y Chile; uno en proceso de admisión, Costa Rica; un actor de importancia global como Brasil en calidad de "socio clave" ; un programa específico de país para el Perú y sucesivos planes acción con el Gobierno de Argentina.

El programa regional de cooperación (como la actuación general de la OCDE en el mundo) dice promover las mejores prácticas de gobierno, que permitan el aumento de la productividad y el crecimiento económico de Latinoamérica, al tiempo que integren una orientación “inclusiva”. Los tres ejes del programa regional son "gobernanza, productividad e inclusión social" y se enmarcan en la apuesta general de la Organización por promover el uso y aplicación de sus estándares regulatorios y de políticas a través del ciclo: (a) estadísticas comparables; (b) evaluación; (c) diálogo; y (d) asesoramiento sobre políticas (OCDE, 2017).

Asimismo, la Organización se ha abierto a un mayor rango de materias de interés, bajo una extensión de los asuntos del trabajo de décadas como economía, energía, desarrollo, capital y políticas de inversión, a otros que considera relacionados, en tanto que harían posible la paz, la democracia liberal y el adecuado funcionamiento de economías de mercado. De manera que ahora merecen atención de la ocDE asuntos como educación, empleo, ciencia, tecnología, ambiente, entre otros, y se procura la convergencia de políticas en estas temáticas (Wolfe, 2008).

Salzman (2005) describe entonces a la ocDE como una institución internacional que "lejos de ser una reliquia de la Guerra Fría”, se ha constituido en "una amalgama entre un club de países ricos, una firma consultora para gobiernos en materia de administración y un cuerpo legislativo", que "ofrece un foro restringido para asuntos virtualmente ilimitados" (p. 192).

\section{TAREAS Y MEDIOS UTILIZADOS}

En primer lugar, en la actualidad la ocDE puede ser definida como una organización que "crea, provee y legitima ideas y las ideas cumplen un papel muy importante en la gobernanza global contemporánea (Mahon \& McBride, 2008, 2009), en cuanto esta supone el interés de Estados,

5 El Consejo Ministerial de la ocDE aprobó en el 2007 una resolución para fortalecer los lazos de cooperación, como "socios clave", con Brasil, China, India, Indonesia y Sudáfrica (ocDE, 2007). 
actores no estatales e instituciones internacionales en sumar esfuerzos para enfrentar desafíos que se perciben como compartidos, dada la ausencia (e inconveniencia) de un gobierno de naturaleza global.

En particular, en materia de creación de ideas, la ocDe se ha posicionado como una institución reconocida por su capacidad investigativa y técnica, gracias a su habilidad para atraer expertos sectoriales al diálogo que promueve en las redes de gobernanza sobre las que tiene influencia ${ }^{6}$. Asimismo, la ocDE cuenta con una posición privilegiada en materia de información, dada la habilidad que ha desarrollado para recopilar material cuantitativo comparable acerca del desempeño en diversas materias, tanto de países miembros de la ocDE, como de aquellos que se han interesado en participar de sus actividades.

Las ideas así formadas imprimen fuerza política, cultural y social a las normas y estándares que circulan internacionalmente en cuanto a lo que las administraciones públicas, sociedades y sujetos deben ser. Dichas ideas se materializan, entre otros, en las cerca de 250 publicaciones anuales que produce esta organización, las cuales se divulgan en su portal, en las diversas redes de gobernanza que articula, así como en el amplio número de encuentros que coorganiza en diversos lugares del planeta, en los que reúne anualmente a más de 40.000 funcionarios oficiales y expertos.

En segunda instancia, de la mano de la promoción y circulación de ideas, en el contexto de "gobernanza sin gobierno global", la ocDE se ha comprometido de forma cada vez más evidente durante las últimas décadas en la construcción de estándares, principalmente en la forma de soft $l a w^{7}$, que resultan de la interacción de actores convocados en las

\footnotetext{
6 Las redes de gobernanza constituyen círculos transnacionales de "intercambio", en los que participan actores de diversa naturaleza (estatales y no estatales) y niveles (globales, nacionales y locales). Picciotto (2006) denomina a esos círculos "redes regulatorias globales" o "gobernanza en red" (network governance), término también utilizado por Tan (2011) para referirse al mismo fenómeno. Por otro lado, Slaughter (2004) las denomina redes de gobierno (government networks), mientras que Stone (2001) se refiere a ellas como redes globales de políticas públicas ( global public policy networks, GPPNS). Esta diversidad de expresiones alude a formas contemporáneas de ejercicio del poder a nivel global, con importantes consecuencias en la producción y circulación global de marcos regulatorios y modelos de políticas públicas. Slaugther y Stone coinciden en que una característica central de este tipo de espacios consiste en la identificación de un problema compartido, respecto del cual se propicia un intercambio de información, conocimiento, buenas prácticas, lineamientos, alternativas de regulación, políticas públicas y discursos (Stone, 2004). Algunos ejemplos de redes son el Centro de Estudios Monetarios Latinoamericanos (CEMLA), la Red de integridad pública del BID-OCDE en América Latina y El Caribe, la Red latinoamericana y del caribe de mejora regulatoria, así como la International Network on Financial Education (INFE). 7 O derecho internacional blando, aunque no sea jurídicamente vinculante, ejerce una significativa influencia sobre agencias gubernamentales en la incorporación de marcos regulatorios que circulan globalmente y en la transferencia de políticas. Al respecto puede consultar en Chinkin (1989), Weiss (2015) y Dunoff et al. (2010).
} 
diversas redes que auspicia y a las que ofrece apoyo (Porter \& Webb, 2008; Woodward, 2008) ${ }^{8}$.

En este sentido, la OCDE cuenta con cerca de 245 instrumentos jurídicos internacionales (Organization for Economic Co-operation and Development, 2018) que incluyen "decisiones, recomendaciones, declaraciones, acuerdos internacionales y arreglos" desarrollados en el marco de la labor de su organización (OECD, 2013, párr. 17). Dichos estándares ejercen una importante influencia no solo respecto de los Estados miembros de la Organización, sino también en relación con aquellos que se encuentran en proceso de admisión, o que mantienen con ella alguna relación de cooperación.

Por ejemplo, en el caso de un Estado en proceso de admisión ${ }^{9}$, este debe declarar si (a) acepta, (b) acepta y solicita un periodo de implementación, (c) acepta bajo reservas u observaciones o (d) rechaza, los estándares jurídicos de la ocDE. Por supuesto, la oCDE espera que los países que aspiran a ser miembros utilicen lo menos posible la aceptación bajo reservas u observaciones, así como los rechazos de las disposiciones que ha adoptado la Organización. Así mismo, la idea es que el Estado examinado justifique adecuadamente cualquier decisión diferente de la aceptación sin condiciones, la cual puede ser materia de discusión con los comités de revisión en el marco del proceso de adhesión (OECD, 2013, párr. 18).

En tercer lugar, la oCDE juega un papel destacado en cuanto a promover procesos de socialización entre Estados, partes de Estados, actores privados, firmas consultoras y ONG, entes a los que convoca permanentemente en redes, así como todo tipo de espacios de intercambio, foros y encuentros de expertos, en los que se discuten las "mejores" experiencias de gobierno, marcos regulatorios y políticas públicas. De esta manera, la ocDE cumple un importante rol en la conformación y proliferación de redes de gobernanza (Salzman, 2005; Slaugther, 2004), a través de las cuales promueve (pero también define y canaliza) debates globales acerca de los marcos regulatorios y

\footnotetext{
8 Así, los estándares internacionales y las ideas que los acompañan contribuyen a determinar qué debe ser un Estado moderno (al modelar sus comportamientos internos y externos), así como a "identificar problemas" y "mapear las mejores prácticas" para su resolución, de forma que los esfuerzos se dirijan en esa dirección (Mahon \& McBride, 2008).

9 Proceso por el que pasó el Estado colombiano desde enero del 2011, cuando el Gobierno de Juan Manuel Santos anunció su intención de promover la admisión de este país en la Organización. Lo cual se materializó en un acuerdo entre el Estado colombiano y la ocDE, que fue firmado el 30 de mayo del 2018, aprobado por el Congreso de la República mediante la Ley 1950 del 2019 y avalado por la Corte Constitucional en sentencia C-492 del 2019.
} 
las políticas públicas más urgentes para las sociedades en el contexto de la globalización ${ }^{10}$.

En cuarto lugar, como se verá, la OCDE juega un papel simbólico de distribución y asignación de prestigio a los Estados que siguen sus lineamientos, se someten a sus exámenes y se encuentran en los mejores lugares de sus rankings. La capacidad de ejercer esta función le es atribuida a partir de la autoridad que se otorga a su actuación (pues sus recomendaciones y evaluaciones se apoyan presuntamente en conocimiento experto, construido a partir de investigaciones y evidencias comprobables), a su papel en la identificación de las "mejores prácticas de gobierno" y al carácter limitado (casi exclusivo) de su membresía.

La OCDE cuenta con un "sello de calidad", que le confiere la potestad de certificar a quienes mejor se han articulado a las tendencias generales y condiciones de la globalización contemporánea. En este sentido, la Organización administra una importante idea en el marco de la interacción entre actores en el plano internacional, aquella que asocia prestigio y reconocimiento con los Estados que han interiorizado e implementado cotidianamente sus valores, métricas y marcos de actuación ${ }^{11}$.

En consecuencia, este "club de naciones ricas", como ya es usual denominar a la OCDE en todo tipo de declaraciones oficiales y literatura (Mahon \& McBride, 2008. 2009), constituye un lugar privilegiado para

\footnotetext{
10 "La OCDE es huésped por excelencia de las redes regulatorias transgubernamentales, así como un catalizador para su creación. Su principal función, por lo menos en las últimas décadas, ha sido convocar a oficiales de gobiernos en temas específicos para determinar las mejores formas de resolver problemas económicos o regulatorios comunes y en algunas ocasiones para promulgar un código modelo para su solución" (Slaugther, 2004, p. 46).

11 Mahon y McBride (2008) señalan que la oCDE habitualmente tiene a su alcance dos tipos de medios de incidencia respecto de las preferencias y decisiones de los Estados, que corresponden precisamente a las formas de operación del poder en el marco de la mencionada gobernanza: "La ausencia de una jerarquía formal, en cambio, sugiere la utilización de regulación 'blanda' [soft] y 'dura' $[$ hard $]$ (por ejemplo, normas formales y directivas). Entonces, además de la regulación clásica, con normas formales y directivas respaldadas de sanciones por violación, el emergente sistema de gobernanza transnacional incluye modos inquisitivos y meditativos de regulación (Jacobsson 2006), en los que la ocDE se encuentra altamente involucrada. La regulación inquisitiva incluye la supervisión o el monitoreo de las acciones de los Estados: 'los Estados miembros no están obligados a seguir políticas específicas, pero se les pide abrir lo que están haciendo al examen y juicio críticos' [...] Igualmente, prácticas como el establecimiento de señales de progreso [benchmarks] y la organización de procesos de revisión por pares suponen la auditoría, comparación y ranking de las prácticas estatales. En contraste, las actividades meditativas 'son principalmente comprendidas como discusiones entre expertos acerca de cuál es la mejor forma o formas de hacer algo'” (p. 6). Estos medios de actuación de la ocDE se hacen más evidentes si se advierte la capacidad e inclinación desarrollada por esa organización para comprometerse en la construcción de redes de gobierno. En particular, la ocDE se ha posicionado como una organización internacional especializada en desarrollar redes que procuran el intercambio entre Estados, partes de Estados, actores no estatales, instituciones internacionales y expertos, alrededor de las mejores prácticas en materia de políticas públicas y marcos regulatorios.
} 
la circulación de marcos regulatorios e ideas sobre la solución de los problemas que, al parecer de forma más o menos semejante, enfrentan las sociedades. A diferencia de otras organizaciones internacionales, la membresía en la ocDE es más reducida, en cuanto convoca a los Estados más seriamente comprometidos con la "economía de mercado y la democracia liberal" (Mahon \& McBride, 2008). Es, en estos términos, un importante (pero poco advertido hasta ahora) nodo de globalización (globalization hub), que se concentra en la transferencia de políticas (policy transfer) como recomendaciones sobre buenas prácticas y en el aprendizaje sobre ellas (policy learning) (Mahon \& McBride, 2008).

Para Wolfe (2008), la gran contribución de la ocDE radica en su capacidad para influir en las políticas públicas mediante "la identificación de normas y principios para las negociaciones que tienen lugar en otras organizaciones internacionales que usan sus ideas, especialmente el G8 y la OMc" (p. 41). En la actualidad, más que procurar una acción internacional conjunta sobre los desafíos globales, la ocDE le apuesta a la convergencia y coordinación de políticas nacionales, bajo la consolidación de lo que algunos de sus funcionarios denominan una "cultura común de políticas" (common policy culture) (Wolfe, 2008; Leimgruber \& Schmelzer, 2017).

\section{LECTURA DE LA EVIDENGIA}

Aunque uno de los pilares del trabajo de la OCDE consiste en la transferencia de marcos regulatorios y de políticas, mediante el "aprendizaje" y la circulación del conocimiento experto (Mahon \& McBride, 2009) -como se indica en cualquier documento de esta institución y en muchos análisis sobre las premisas que fundan su labor-, lo cierto es que muy a menudo se ha puesto en duda la capacidad de la organización para tomarse en serio la evidencia existente y construir recomendaciones sobre hechos comprobables. Es decir, la habilidad de una institución a la que se confiere autoridad a propósito de su presunta trayectoria "analítica", experiencia técnica y competencia investigativa, se pone en entredicho cuando se examina de cerca el fundamento de sus recomendaciones ${ }^{12}$.

12 "La importancia de la oCDE como organización internacional se basa principalmente en su pericia técnica. Es más conocida por sus actividades de investigación [...] Estas actividades de investigación tienen importantes impactos, más allá de la organización de la información. Al reunir a expertos, la ocDE crea redes internacionales. En el papel de identificador y solucionador 
Como lo anotan Leimgruber y Schmelzer (2017), las deficiencias empíricas en los hallazgos y recomendaciones de la ocDE parecen encontrar explicación en su trayectoria histórica y en su interés por presentarse a sí misma como organización experta. Entre 1950 y 1960, la OCDE pasó de ser una institución con una "reputación estadística "notoriamente mala" a convertirse "en un centro para la recolección, armonización y presentación de estadísticas neutrales y confiables” (p. 46).

De esta manera, no es extraño encontrar que muchas de las orientaciones de la OCDE, construidas desde las redes de diálogo, intercambio, aprendizaje y conocimiento en las que juega un papel central, o a partir del trabajo de expertos a los que la Organización integra en su labor, tienen al final un sustento más bien cuestionable. Es posible entonces que muchas de las premisas sobre las que se construyen las recomendaciones de política, provengan de estudios "no conclusivos" o se promuevan sobre la base de un carácter técnico, que es solo aparente. Porter y Webb (2008) presentan este punto en los siguientes términos, al hacer referencia a los principios sobre gobierno corporativo promovidos por la OCDE:

El grado en que los principios no son expresión de un consenso basado en evidencia experta es manifiesta no sólo en el importante papel del juicio en la metodología o en los conflictos políticos que son manejados y suprimidos al no reconocerlos en la formulación e implementación de los principios, sino también en la falta de evidencia científica que sustente el modelo que promueven los principios [...] las afirmaciones que indican que las prácticas de gobernanza corporativa producen resultados económicos positivos 'aún tienen que pasar la prueba de un análisis empírico conclusivo’ [...]

Bajo una observación más cercana entonces, el papel de la ocDE ha sido reforzar tendencias que ya se presentan en los mercados globales y usar su autoridad para presentar un problema potencialmente político y conflictivo como una buena práctica (p. 56).

de problemas transgubernamentales, la ocDE tiene la capacidad en algunos casos de crear el debate y en muchos otros de canalizarlo, fijando los contornos de la discusión sobre lo que es deseable y lo que es posible. El efecto neto puede ser una forma sutil pero significativa de advocacy. Como ha observado David Trubek, el resultado es una 'organización construida en torno a la idea de experticia técnica que ha tenido que enfrentar el hecho de que lo técnico es político[...] podría afirmarse que este trabajo es tan, sino más, importante que la labor formal de producir normas'” (Salzman, 2005, p. 219). 
Siems y Álvarez-Macotela (2017) llegan exactamente a la misma conclusión en cuanto a estos principios, en su versión del 2015, al observar que,

una posible línea de crítica puede ser que las fallas en el gobierno corporativo no fueron las principales razones de la crisis financiera del 2008 y que la investigación empírica sobre si el gobierno corporativo importa en el desarrollo financiero no ha producido resultados inequívocos (p. 18).

De otro lado, en cuanto a las estrategias de generación de empleo promovidas por la OCDE entre 1990 y el 2000, se cuestionó en su momento la paradójica "incapacidad para aprender" a partir de la evidencia existente que presenta la Organización. A pesar de la ausencia de evidencia empírica que respaldara de forma concluyente su apuesta de 1994 por una aproximación neoliberal a las políticas de empleo, y a pesar del buen comportamiento durante la década posterior de los países que no acataron completamente la receta de la Organización, esta insistió en el 2006 en privilegiar su lectura del problema (McBride et al., 2008) ${ }^{13}$.

En la revisión del 2006 de los términos de su política, la OCDE reconoció la existencia de enfoques alternativos en cuanto a las estrategias de empleo, al tiempo que procuró desacreditarlos por todos los medios, para advertir que los "generosos" esquemas de beneficios sociales que conservan algunos países, ocultan problemas más extendidos de desempleo y constituyen una política riesgosa (McBride et al., 2008; Jackson, 2008; Mahon \& McBride, 2009). Contra toda evidencia, la ocDE desaconseja la idea de mantener los niveles de gasto público de algunos países en el otorgamiento de beneficios sociales y protección al trabajo, e insiste en la "buena práctica" de reducir todas las condiciones que generen rigideces en el mercado laboral (McBride et al., 2008; Jackson, 2008; Mahon \& McBride, 2009).

En materia educativa también se ha cuestionado que la ocDE presente una agenda de políticas sobre una base técnica, cuando realmente se sustenta en una "lectura ideológica", "diametralmente

13 Desde la década de 1990, la ocDE recomienda una serie de buenas prácticas relacionadas con la eliminación de factores que puedan generar rigideces en los mercados laborales y que supongan una excesiva intervención del Estado. Las estrategias sugeridas por la ocDE (un total de ochenta medidas) se orientan a la "flexibilización laboral", la desregulación y la reducción de la influencia de factores como sindicalización, beneficios sociales y protección al trabajo (McBride et al., 2008). 
opuesta de las lecciones de política" que resultan de los datos y hallazgos existentes (Rubenson, 2008). En este campo, las orientaciones de la Organización se han dirigido a recomendar limitaciones del gasto público, con un consecuente aumento del gasto privado de empresas, familias e individuos (Rubenson, 2008).

Se privilegia así la construcción y divulgación de ciertas "buenas prácticas”, en particular, aquellas que revelan austeridad fiscal, reducción de gasto público, gasto de individuos y familias en la adquisición de servicios sociales. Al mismo tiempo, se excluyen las experiencias exitosas que revelarían las virtudes opuestas.

De esta manera, la capacidad investigativa y analítica de la OCDE, así como la autoridad que se desprende de ella, no deja de ser una afirmación de principio, con propósitos retóricos y con enormes efectos políticos. De las altamente apreciadas fortalezas intelectuales de la oCDE, se deriva en buena medida la importancia que se asigna globalmente a las recomendaciones sobre buenas prácticas que organiza, sistematiza e impulsa esa institución. La imagen que ha construido a menudo le permite dotar a sus recomendaciones de un aura de experticia incontestable, no siempre fundada en evidencia comprobable, en estudios conclusivos y en análisis alejados de cualquier orientación política.

\section{¿ENTRE NEOLIBERALISMO Y (NEO) LIBERALISMO INCLUSIVO?}

La orientación general de la OCDE con su idea de una estructura estatal amigable para los mercados, insistencia en la reducción del gasto público en la satisfacción de necesidades ciudadanas, promoción de reformas institucionales que privilegiaran la eficiencia, énfasis tanto en el monetarismo como en la ortodoxia financiera y, en general, su comprensión del ideal de Estado moderno, ha estado determinada por una racionalidad neoliberal, al menos entre la mitad de la década de 1970, hasta la mitad de los 1990 y comienzos de los 2000.

En efecto, la publicación del informe McCracken en 1977 sobre la relación entre la estabilidad de precios y el pleno empleo, representó el momento exacto en el que el neoliberalismo se posicionó como la orientación manifiesta de la ocDE (Mahon \& McBride, 2008; Wolfe, 
$2008)^{14}$. Este informe ofrecía una interpretación de los problemas

que en ese entonces enfrentaban las economías capitalistas ${ }^{15}$, hacía recomendaciones para su solución en la forma de un inevitable y estrecho "margen de acción" (narrow path), y observaba la necesidad de un cambio de naturaleza política ${ }^{16}$.

En este último aspecto, como lo señalaba Keohane (1978), el reporte sugería que "en nombre de la prosperidad económica [...] los Estados democráticos modernos ejerzan mayor disciplina respecto de sus economías, al tiempo que resistan la tentación de otorgar beneficios de corto plazo a sus ciudadanos mediante un gasto público extendido" (Keohane, 1978, p. 109). Sin mayores evidencias, el informe McCracken señala que una de las principales causas del aumento de la inflación consistía en el "rápido aumento" de "aspiraciones populares" y de la negociación colectiva del aumento de salarios (Keohane, 1978, p. 113). El reporte señalaba expresamente que "el creciente interés público en objetivos relacionados con distribución del ingreso y el bienestar de las poblaciones de países en desarrollo, ha alterado las metas económicas y ha llevado a presiones por una mayor intervención estatal y administrativa en los asuntos económicos" (Keohane, 1978, p. 113):

14 La OCDE nombró a ocho importantes economistas para la elaboración de dicho informe, con el fin de abordar "las cuestiones de políticas involucradas en la búsqueda, por los Estados miembros, de crecimiento no inflacionario y altos niveles de empleo en el marco de cambios estructurales" (Keohane, 2008, p. 108). El nombre con el que fue ampliamente conocido el informe se debe al jefe del equipo de economistas que participó en su elaboración. Se trataba de Paul McCracken, economista de Harvard, quien había servido como asesor bajo la administración de Richard Nixon y era cofundador del American Enterprise Institute (Gayon, 2012), un think tank de orientación conservadora, que se propone defender y mejorar los principios de la "democracia capitalista", entre otros, "un gobierno limitado, la iniciativa privada, la libertad y responsabilidad individuales" (American Enterprise Institute, 2009).

15 El de mayor atención en el informe era el estancamiento económico con inflación. 16 Más allá de los detalles del informe, este generó críticas en una célebre revisión del politólogo Robert Keohane en 1978, quien básicamente se lamentaba de la falta de evidencia sobre la cual se fundaban los diagnósticos y las recomendaciones de un reporte al que se le otorgaba tal importancia. Entre otras cosas, Keohane advertía que el informe no ofrecía ninguna evidencia sobre las causas de la situación inflacionaria (de la que se ocupaba principalmente), ni prueba de factores centrales en el diagnóstico, como "expectativas inflacionarias" o "falta de confianza". Keohane señalaba entonces que el reporte McCracken presentaba una "limitada" lectura económica de un problema, que requería una comprensión macroeconómica, política y sociológica de mayor alcance: "Es difícil evitar la conclusión de que el informe McCracken no presenta una explicación convincente y bien especificada de la inflación y la recesión. Las causas fundamentales citadas en el informe, que son políticas, sociológicas y psicológicas, no se describen con precisión ni se explican. El informe no desarrolla un análisis interdisciplinario; de hecho, los autores ni siquiera manifiestan la conciencia de que uno es necesario" (Keohane, 1978, p. 115). Esta situación corresponde a la inclinación de la ocDE ya mencionada, a recomendar políticas y decisiones sobre la base de una lectura ideológica o, al menos, bastante parcial de los problemas y de las alternativas de solución al alcance de los gobiernos. 
El profesor McCracken y sus colegas no se contentan simplemente con señalar los dilemas que el capitalismo plantea para la democracia. Por el contrario, defienden enérgicamente políticas pro-capitalistas, utilizando supuestos y valores conservadores no sometidos a examen para plantear su punto. Aunque no emprenden un análisis sofisticado de las instituciones y los procesos políticos y sociales, están dispuestos a hacer recomendaciones de política que descansan en una ideología política y en un alegato económico. Así, el informe muestra un sesgo consistente en contra del aumento de la participación estatal en la economía, en particular mediante una mayor propiedad pública o controles salariales y de precios (Keohane, 1978, p. 118).

De esta forma, es posible afirmar la existencia de una tradición de compromiso de la ocDE con una agenda neoliberal, debido a su inclinación a privilegiar la austeridad fiscal, la privatización de servicios, la presión por la liberalización del comercio internacional, entre otros, que se posicionó de forma franca y abierta desde la mitad de la década de 1970, hasta mediados y fines de la década de 1990. Como afirmó en un momento determinado un antiguo funcionario de la organización, "la ocDE es a la economía clásica lo que San Pedro es a la cristiandad, es decir, es quien guarda las llaves" (Porter \& Webb, 2008, p. 46).

A pesar de esta orientación general, que marca en buena medida hasta la actualidad su comprensión del Estado y de las actuaciones en las que este debe comprometerse, la ocDE no es uni-dimensional. Las "ideas dominantes en los departamentos económicos" (de una mayor inclinación neoliberal), no necesariamente son compartidas por otras áreas que se ocupan de políticas sectoriales de empleo, educación, ambiente, seguridad social, entre otras. La agenda neoliberal de la ocDE dista de ser completamente coherente y puede encontrarse con discursos, incluso algunos promovidos por esa organización internacional, que la retan o cuestionan, al menos parcialmente. La Organización tendría hoy en día una aproximación más diversa a una serie de materias, que se movería entre el neoliberalismo y un "liberalismo inclusivo" o "liberalismo innovador". Esto le permitiría interesarse por asuntos relativos a la superación de la pobreza y la desigualdad (Mahon \& McBride, 2008, 2009; Porter \& Webb, 2008; Pal, 2008) ${ }^{17}$. Se trataría en realidad de un giro desde una perspectiva francamente neoliberal de ajuste estructural, a una 
aproximación más "suave", con atención a la pobreza, la participación y la "buena gobernanza” (Walker, 2008).

Sin embargo, al menos dos razones permiten dudar del impulso inclusivo de la Organización. De un lado, la innegable influencia que han ejercido los departamentos económicos, y en general la economía como disciplina sobre el conjunto de la OCDE, entre otras razones, por el destacado papel que juegan de acuerdo con los propósitos generales de la institución y, en particular, en el marco del examen de las políticas nacionales (Leimgruber \& Schmelzer, 2017). Por otro lado, como lo observa Ruckert (2008), la OCDE reconoce cierto margen de decisión en las instituciones nacionales y muestra mayor interés por una agenda inclusiva, sin dejar de recordar que los Estados deberían seguir políticas macroeconómicas y financieras "apropiadas", considerando por supuesto como tales, aquellas que más correspondan o se acerquen al "conocimiento técnico" y a las "buenas prácticas" construidas en los espacios de expertos y en las redes que la ocDE despliega para el diálogo global:

Lo que es ciertamente problemático de este emergente consenso neoliberal inclusivo, es que las preocupaciones por la inclusión siguen estando circunscritas a un marco macroeconómico que no se aleja sustancialmente de ideales neoliberales anteriores (Ruckert, 2008, p. 107).

En ese marco, a comienzos de la década del 2000 se empieza a gestar un movimiento "inclusivo" en las orientaciones de la OCDE en los sectores más directamente relacionados con el bienestar social de los ciudadanos, como salud y trabajo, o materias como desarrollo infantil e igualdad de género, sin abandonar el contexto general de direccionamiento hacia un marco macroeconómico que privilegia el control del gasto público, la flexibilización laboral, la privatización de servicios y la eliminación de barreras al comercio ${ }^{18}$.

18 Mahon y McBride dudan sobre la existencia de un cambio significativo en el conjunto de la Organización y se preguntan hasta qué punto esto "representa un nuevo paradigma, distinto del neoliberalismo que ha caracterizado a las aproximaciones de política de la ocDE desde la mitad de la década de los 70, o si representa un ajuste, modificación o afinamiento de ese enfoque, como respuesta a las críticas al modelo neoliberal, sin sacrificar los fundamentos de esa aproximación, ofreciendo lo que Graefe (2006) llama 'mecanismos de flanqueo' [flanking mechaisms]" (Mahon \& McBride, 2008, p. 18). En este sentido, mientras se espera que los Estados se comprometan en políticas, estrategias y medidas para la reducción de la pobreza, apoyadas 


\section{UN IDEAL DE ESTADO Y DE SUJETO}

La orientación general de la OCDE, con sus eventuales matices y tensiones, se materializa en la comprensión de lo que debe ser el comportamiento colectivo e individual de las poblaciones. Así, el Estado y los sujetos constituyen aspectos de especial atención para sus análisis y recomendaciones.

En primer lugar, la oCDE juega un importante papel en la formación de redes de conocimiento, y a través de estas, desempeña un papel decisivo en la construcción de las identidades de los Estados. Las conclusiones de Porter y Webb (2008), a partir del análisis de las recomendaciones de la oCDE sobre gobierno corporativo, revelan que la Organización contribuye a la definición de una suerte de identidad, basada en una idea difusa de liderazgo y superioridad, que muchos Estados, tanto miembros como no miembros están interesados en obtener y retener.

Lo anterior se acompaña de la capacidad de la OCDE, dado el prestigio que se le confiere en materia de marcos regulatorios y políticas públicas, de presentar ciertas prácticas relativas al funcionamiento de los mercados como "no problemáticas, apolíticas, y relativamente rutinarias formas de hacer las cosas, que son conocidas por ser las mejores, dada la apariencia de consenso que la ocDE crea" (Porter \& Webb, 2008, p. 57). En este sentido, la oCDE juega entonces un papel destacado en la construcción de un sentido común acerca de lo que es normal, sensato, práctico y deseable en el desarrollo de políticas y prácticas institucionales sobre el gobierno de las poblaciones.

Esto se logra a través de los medios de operación de la OCDE explicados previamente: la construcción de estándares sobre la base de la identificación de las mejores prácticas de gobierno, la difusión de los mismos a través de redes de gobernanza, la determinación de indicadores, la elaboración de rankings, la interacción entre "constelaciones de actores privados y públicos" de diversa naturaleza y nivel, la apertura del Estado a la revisión por pares de su performance y prácticas administrativas cotidianas, así como de la socialización entre funcionarios públicos en

en una comprensión local y participativa del problema, las expectativas de las instituciones internacionales se mantienen en la forma de "recomendaciones" sobre un gasto público "prudente y antiinflacionario", aumento de los niveles de recaudación tributaria, liberalización del comercio, privatización de servicios públicos (Ruckert, 2008) y flexibilización laboral (McBride et al., 2008; Jackson, 2008). 
un amplísimo listado de eventos y reuniones convocadas por esa misma organización, en alianza con diversas instituciones internacionales ${ }^{19}$.

A partir de actividades como las mencionadas, la OCDE participa de un proceso global de determinación de las expectativas que caracterizan qué significa un Estado moderno. En efecto, “define estándares sobre el comportamiento adecuado para Estados que buscan identificarse como modernos, liberales, amigables con los mercados (market friendly) y eficientes" (Porter \& Webb, 2008, p. 44). Se trata de una "lógica de lo apropiado" que en algún sentido señala una corrección, un ideal de ser, que se espera de las administraciones públicas que quieren ser consideradas respetables y que como tales estén en condiciones de atraer inversiones, capitales y, en general, atención de actores de otras partes del planeta. En una palabra, lo anterior supone en última instancia una sofisticada disciplina, en la que la ocDE juega un papel central como facilitador y árbitro en la circulación de ideas, así como en la construcción de reglas y parámetros conforme a los cuales se valora a los Estados.

En segundo lugar, esta comprensión del Estado se desdobla y reproduce en la racionalidad rectora que orienta la formación de los sujetos. Por supuesto no se sugiere una correspondencia absoluta, pero sí se observa una cierta convergencia, de manera que el ideal de Estado moderno se acompaña de un ideal de "capital humano".

En este sentido, la oCDE ha llegado a controlar en muy buena medida la orientación global de las políticas educativas (al punto de desplazar a la Unesco) mediante "la construcción de un 'sentido común' de sociedad" (Rubenson, 2008, p. 242), y de una estrecha relación entre las esferas económicas y educativas (Walker, 2008) ${ }^{20}$. En particular, mediante el desarrollo e implementación de indicadores para el seguimiento del

19 "La paradoja es que esta forma de dinámica y desarticulada de interacción de actores y organizaciones, en la formación de reglas suaves y estrategias no coercitivas de monitoreo y cumplimiento, es difusa, confusa y de geometría variable, y aun así crea orden. Este orden es frágil, a diferencia de las jerarquías de mando y control respaldadas por la fuerza y las sanciones, de manera que tiene que ser constantemente recreado y recalibrado. La ocDE es una importante contribuidora a la gobernanza global que ocurre sin un gobierno global. Como espacio y convocante, la oCDE conecta y reconecta incesantemente funcionarios de gobierno alrededor del mundo, al lado de expertos, organizaciones no gubernamentales, y negocios" (Pal, 2008, p. 74). 20 No obstante que en el tratado constitutivo de la OCDE no se prevé la educación como parte de su marco de acción misional (con excepción de una alusión marginal al entrenamiento vocacional), está institución ha desarrollado a lo largo de su existencia un creciente interés e influencia en el análisis y la construcción de estándares internacionales en materia de desempeño de los sistemas educativos nacionales, tanto respecto de la educación básica y media, como de la terciaria y superior. Esta tendencia se apoya por supuesto en el contexto de la labor general de la oCDE que involucra la promoción del desarrollo y bienestar de los ciudadanos. 
desempeño de los estudiantes y de los sistemas educativos, la OCDE ha procurado articular logros educativos con resultados económicos.

El cambio de orientación de la ocDE en la década de 1970 en términos de política económica, se reprodujo en una modificación de los contenidos e inclinaciones de su agenda educativa. Hasta entonces, la apuesta debía consistir en procurar el acceso más extendido posible a una educación gratuita o al menos de bajo costo para el ciudadano. A partir de la década de 1970 y en el contexto del creciente interés de la organización por los mercados de trabajo, la aproximación a la educación cambió. De la atención al funcionamiento de los sistemas educativos como bienes públicos, se comenzó a posicionar un énfasis en la educación para el "mundo del trabajo" y como factor de producción ${ }^{21}$.

Este giro en la agenda sectorial de la ocDE supuso un creciente interés por la reevaluación del papel de la educación, de manera que esta diera respuesta a las necesidades de la competencia económica, garantizara una mayor adaptación de las personas a los mercados de trabajo, permitiera el involucramiento del sector privado, así como una relación más estrecha entre establecimientos educativos y empresas empleadoras ${ }^{22}$.

A lo anterior se suma el papel central que ha jugado la ocDE en la construcción de una convergencia global en materia de educación financiera, en particular, a partir de la crisis de los mercados bursátiles del 2008, a través de la Red Internacional sobre Educación Financiera (INFE, por sus siglas en inglés), creada y auspiciada por la oCDE (G20-OECD, 2013; G20, 2012). Conforme a los estándares de la OCDE y del G20 en materia de educación financiera, resulta imperativo que los sujetos aprendan a

21 "El nuevo imperativo de política económica rompió el amplio consenso sobre las políticas educativas que había caracterizado la década de los 60 y 70. Dentro del nuevo imperativo económico, la relación entre la educación y la economía, particularmente el trabajo, se convirtió en el asunto clave, y surgió una gran batalla entre ideologías e intereses en competencia. La ocde no hizo más llamados a la expansión general de la educación pública, sino que quiso drásticas reformas a la naturaleza de ésta. Las preocupaciones por la igualdad de oportunidades fueron reemplazadas por llamados a la flexibilidad y sensibilidad a las necesidades del mercado de trabajo [...] La educación ya no era promovida como un bien común sino como un instrumento en la competencia global [...] El esfuerzo cambió hacia el papel de la educación en el desarrollo de la capacidad individual para el aprendizaje continuo, la creatividad y la autosuficiencia, promoviendo de este modo su calidad y flexibilidad para el mercado de trabajo" (Rubenson, 2008, p. 253). 22 Igualmente, este giro implicó el uso de la noción de "aprendizaje a lo largo de la vida" (lifelong learning, reedición del concepto de educación recurrente), que supone un proceso permanente "no necesariamente intencional ni estructurado", que puede encontrarse en "instituciones formales e informales", tanto dentro de la escuela como fuera de ella (por ejemplo, con mayor atención al aprendizaje en el trabajo), que puede ser dirigido o autodirigido y que, como su nombre lo indica, se extiende durante toda la existencia de los "recursos humanos" (Rubenson, 2008, p. 256). Esta apuesta supone un desplazamiento del interés por las instituciones y la visión más general del sistema educativo, hacia un énfasis en el individuo, hacia la motivación y capacidad para desarrollar su "propio proceso de aprendizaje" (Rubenson, 2008; Walker, 2008). 
actuar conforme a criterios corporativos, que les permitan fortalecer su portafolio, atraer inversiones, emprender, manejar riesgos financieros, invertir de forma adecuada y planear financieramente toda su existencia (Arthur, 2012; Asobancaria, 2014a, 2014b; G20, 2012, 2011). Entre otros medios, esta formación debería ser ofrecida desde la edad más temprana posible a través de la inclusión de contenidos curriculares en la educación básica formal (OECD, 2005; OCDE-INFE, 2015) ${ }^{23}$.

En consecuencia, la racionalidad rectora que ha orientado de manera general la actuación de la OCDE, se materializa en la promoción global de una subjetividad específica. Bajo la convergencia global sobre la educación financiera y a propósito del papel destacado de la organización en materia educativa, se promueve el ideal de un sujeto que se ve obligado a afirmarse como un capital humano, que debe usar mecanismos del mercado financiero para aumentar su valor y procurar el logro de sus objetivos. Este sujeto debe gestionar de forma individual, con el apoyo del sector financiero y asegurador, los diferentes riesgos de la vida, entre ellos, invalidez, vejez, muerte y desempleo, ante la renuncia a una apuesta colectiva, basada en criterios de solidaridad para la gestión de esas contingencias.

\section{REFERENCIAS}

American Enterprise Institute of Public Policy Research. (2009). AEI's Organization and Purposes. https://web.archive.org/web/20090212000018/

Arthur, C. (2012). Financial Literacy Education: Neoliberalism, the Consumer and the Citizen. Sense Publishers.

Asobancaria. (2014a). Semana económica. Asobancaria.

Asobancaria. (2014b). Programas de educación financiera de los bancos de Colombia. https://www.sabermassermas.com/programas-de-educacion-financiera-delos-bancos-en-colombia/

23 Contrario a lo que sostienen Pal (2008) y Mahon (2010), el énfasis reciente de la ocDE sobre "inversión en desarrollo de capital humano" no supone realmente un distanciamiento de una racionalidad rectora neoliberal. La comprensión de las personas como "capital humano" permite cuestionar la existencia de ese presunto giro, a propósito de un "liberalismo inclusivo". Al contrario, el interés reside en el "capital humano" para la "competitividad y crecimiento económicos", bajo una subjetividad articulada a una comprensión particular del papel del Estado y de los mercados. No porque sea deseable desde un punto de vista de satisfacción de necesidades humanas, de garantía de derechos y libertades humanas o por razones de justicia social y solidaridad. De igual manera, esa apuesta por el gasto público dirigido a la niñez, se encuentra sometida a las directrices más generales que promueve la misma ocDE en materia de ajuste estructural y recorte en la atención de necesidades sociales. 
Chinkin, C. (1989). The Challenge of Soft Law: Development and Change in International Law. The International and Comparative Law Quarterly, 38(4), 850866. https://doi.org/10.1093/iclqaj/38.4.850

Dunoff, J., Ratner, S., \& Wippman, D. (2010). International Law: Norms, Actors, Process. Wolters Kluwer.

G20-oECD. (2013). Advancing National Strategies for Financial Education. https://www. oecd.org/finance/financial-education/advancing-national-strategies-forfinancial-education.htm

G20-OECD. (2017). G20/OECD INFE Report on Adult Financial Literacy in 20 Countries. https://www.oecd.org/finance/g20-oecd-infe-report-adult-financial-literacyin-g20-countries.htm

G20. (2011). G20 High Level Principles on Financial Consumer Protection. https://www. oecd.org/daf/fin/financial-markets/48892010.pdf

G20. (2012). Principios de Alto Nivel de la OCDE/INFE sobre estrategias nacionales de educación financiera. http://finanzasparatodos.es/comun/pdf_varios/5_ PEF_-_Principios_de_Alto_Nivel_sobre_Estrategias_Nacionales_de_ Educacixn_Financiera.pdf

Gayon, V. (2012). The OECD and the "Crisis" of Keynesianism : the McCracken Report, 1975-1980. Paris Dauphine University. https://basepub.dauphine.fr/ handle/123456789/10894

Goodman, R., \& Jinks, D. (2013). Socializing States: Promoting Human Rights Through International Law. Oxford University Press. https://doi.org/10.1093/ acprof:oso/9780199300990.001.0001

Jackson, A. (2008). "Crafting the Conventional Wisdom": The OECD and the Canadian Policy Process. En R. Mahon \& S. McBride, The OECD and Transnational Govervance (pp. 170-187). University of British Columbia.

Keohane. R (1978) Economics, Inflation, and the Role of the State: Political Implications of the McCracken Report, World Politics, Vol. 31, No. 1 (Oct., 1978), pp. 108-128. https://doi.org/10.2307/2009969

Leimgruber, M., \& Schmelzer, M. (2017). From the Marshall Plan to Global Governance: Historical Transformations of the oeEc/oecd, 1948 to Present. En M. Leimgruber \& M. Schmelzer, The OECD and the International Political Economy Since 1948 (pp. 23-61). Palgrave. https://doi.org/10.1007/978-3-31960243-1_2

Mahon, R., \& McBride, S. (2008). The OECD and Transnational Governance. The University of British Columbia.

Mahon, R., \& McBride, S. (2009). Standardizing and disseminating knowledge: the role of the OECD in global governance. European Political Science Review, 1(1), 83-101. https://doi.org/10.1017/S1755773909000058

McBride, S., McNutt, K., \& Williams, R. (2008). Policy Learning? The oecD and its Jobs Strategy. En R. Mahon \& S. McBride, The OECD and Transnational Governance (pp. 152-169). University of British Columbia.

ocDE. (1960). Convención de la Organización de Cooperación y Desarrollo Económicos. http://www.oecd.org/acerca/documentos/convenciondelaocde.htm 
OECD. (2007). OECD Council Resolution on Enlargement and

Enhanced Engagement. https://www.oecd.org/brazil/

oecdcouncilresolutiononenlargementandenhancedengagement.htm

OECD. (2013). Roadmap for the Accession of Colombia to the OECD Convention. https:// one.oecd.org/document/C(2013)110/FINAL/en/pdf

oCDE. (2017). Activos con América Latina y El Caribe. https://issuu.com/oecd.

publishing/docs/activos-con-america-latina-y-el-caribe

OCDE-INFE. (2015). National Strategies for Financial Education: OECD/INFE Policy

Handbook. https://www.oecd.org/daf/fin/financial-education/nationalstrategies-for-financial-education-policy-handbook.htm

Pal, L. (2008). Inversions Without End: the OECD and Global Public Management

Reform. En R. Mahon y S. McBride, The OECD and Transnational Governance (pp. 60-76). University of British Columbia.

Picciotto, S. (2006). Regulatory Networks and Global Governance. En WG Hart Legal Workshop 2006: The Retreat of the State: Challenges to Law and Lawyers, 2006-06-272006-06-29. [Unpublished]. University of London.

Porter, T., \& Webb, M. (2008). Role of the OECD in the Orquestation of Global Knowledge Networks. En R. Mahon \% S. McBride, The OECD and Transnational Governance (pp. 43-59). University of British Columbia.

Rubenson, K. (2008). ofcd Education Policies and World Hegemony. En R.

Mahon, \& S. McBride, The OECD and transnational governance (pp. 242-259).

University of British Columbia.

Ruckert, A. (2008). Making Neo-Gramscian Sense of the Development Assistance

Committee: Towards an Inclusive Neoliberal World Development Order. En

R. Mahon \& S. McBride, The OECD and Transnational Governance (pp. 96-113).

University of British Columbia.

Salzman, J. (2005). Decentralized Administrative Law in the Organization for

Economic Cooperation and Development. Law and Contemporary Problems, 68(3/4), 189-224. https://doi.org/10.2139/ssrn.871757

Siems, M., \& Alvarez-Macotela, O. (2017). The G20/oecd Principles of Corporate

Governance 2015: A Critical Assessment of Their Operation and Impact.

Journal of Business Law, (4), 310-328.

Slaughter, A. M. (2004). A New World Order. Princeton University Press. https://doi. org/10.1515/9781400825998

Stone, D. (2001). Learning Lessons, Policy Transfer and the International

Diffusion of Policy Ideas. Centre for the Study of Globalisation and Regionalisation, Working Paper (69/01).

Stone, D. (2004). Transfer Agents and Global Networks in the

'Transnationalisation' of Policy. Journal of European Public Policy, 11(3), 545-

566. https://doi.org/10.1080/13501760410001694291

Walker, J. (2008). The Inclusion and Construction of the Worthy Citizen Through

Lifelong Learning: A Focus on the OECD. Journal of Education Policy, 24(3),

335-351. https://doi.org/10.1080/02680930802669276 
Weiss, F. (2015). The Device of Soft Law: Some Theoretical Underpinnings.

En F. Weiss \& A. Kammel, The Changing Landscape of Global Financial Governance and the Role of Soft Law (pp. 47-58). Brill Nijhoff. https://doi. org/10.1163/9789004280328_005

Wolfe, R. (2008). From Reconstructing Europe to Constructing Globalization: the oEcD in Historical Perspective. En R. Mahon \& S. McBride, The oECD and Transnational Governance (pp. 25-42). University of British Columbia.

Woodward, R. (2008). Towards Complex Multilateralism? Civil Society and the OECD. En R. Mahon \& S. McBride, The OECD and Transnational Governance (pp. 77-95). University of British Columbia. 